\title{
Finding a New Prognostic Biomarker for Metastatic Colorectal Cancer
}

\author{
Hungdai Kim \\ Department of Surgery, Kangbuk Samsung Hospital, Sungkyunkwan University School of Medicine, Seoul, Korea
}

\section{See Article on Page 315-320}

For detecting new biomarkers in colorectal cancer, whether they are prognostic or predictive, we should understand the mechanism of the metastatic process at the gene expression level. This article presents a new prognostic biomarker, 'placental growth factor (PlGF)' to the clinical field. PlGF, which is a member of the vascular endothelial growth factor (VEGF) family in conjunction with VEGF-A and VEGF-B, is a ligand for VEGF receptor-1, which is expressed on endothelial cells, monocytes/macrophages, and some tumor cells. PlGF was isolated in 1991 from the placenta and was found only in very low levels under physiological conditions, but it was up-regulated in pathological circumstances such as wound healing, ischemia and tumor growth [1].

PlGF up-regulation has been found in human meningiomas, hemangioblastomas, melanomas, and cervical squamous cell carcinomas and is associated with angiogenesis in renal cell carcinomas. On the contrary, PlGF is down-regulated in thyroid carcinomas, germ cell tumors and cervical adenocarcinomas. Chen et al. [2] reported that PlGF was significantly up-regulated in gastric cancer tissue and was significantly correlated with microvessel density-evaluated angiogenesis and tumor stage. In this research, the authors found that the PlGF protein expression level in colorectal cancer tissue was significantly correlated with microvessel density, overall patient survival, and clinicopathological factors such as lymph-node metastasis, tumor stage, and lymphovascular invasion. According to the authors, PlGF seems to be an independent surrogate prognostic factor for colorectal can-

Correspondence to: Hungdai Kim, M.D.

Department of Surgery, Kangbuk Samsung Hospital, Sungkyunkwan

University School of Medicine, 29 Saemunan-ro, Jongno-gu, Seoul 110-746, Korea

Tel: +82-2-2001-2137, Fax: +82-2-2001-2131

E-mail: hungdai.kim@samsung.com

(C) 2012 The Korean Society of Coloproctology

This is an open-access article distributed under the terms of the Creative Commons Attribution NonCommercial License (http://creativecommons.org/licenses/by-nc/3.0) which permits unrestricted noncommercial use, distribution, and reproduction in any medium, provided the original work is properly cited. cer progression. However, for these results to be adopted in the clinical filed, it is very important that a large-sample-size study be brought conducted in a validation setting.

The findings of this research finding suggest that PlGF may constitute a novel approach to colorectal cancer treatment. Several agents against PlGF have already developed or are currently under development. Among these agents, two novel agents against PlGF will be briefly discussed. Firstly, aflibercept (ZALTRAP) is a recombinant fusion protein that acts as a soluble receptor that binds to VEGF-A, VEGF-B and PIGF. In April 2011, Van Cutsem et al. [3] reported that aflibercept improved the primary endpoint of overall survival in the Velour phase III clinical trial for second-line treatment for metastatic colorectal cancer. Secondly, the humanized anti-PlGF mAb (TB-403) is directed against PlGF. A trial with TB-403 has only completed its first phase. Antitumor activity has been demonstrated with TB-403 in human tumor xenograft models of renal cell carcinomas and hepatocellular carcinomas. The mechanism of action for TB-403 is currently under investigation; preclinical studies suggest its effects may include blocking tumor angiogenesis and primary tumor growth, as well as inhibition of metastasis. In addition, PIGF inhibition may complement and potentiate the antitumor effects of VEGF inhibition, in part by inhibiting macrophage recruitment [4].

\section{REFERENCES}

1. Maglione D, Guerriero V, Viglietto G, Delli-Bovi P, Persico MG. Isolation of a human placenta cDNA coding for a protein related to the vascular permeability factor. Proc Natl Acad Sci U S A 1991; 88:9267-71.

2. Chen CN, Hsieh FJ, Cheng YM, Cheng WF, Su YN, Chang KJ, et al. The significance of placenta growth factor in angiogenesis and clinical outcome of human gastric cancer. Cancer Lett 2004;213: 73-82.

3. Van Cutsem E, Tabernero J, Lakomy R, Prausova J, Ruff P, Van Hazel G, et al. Intravenous (IF) aflibercept versus placebo in combination with irinotecan/5-FU (FOLFIRI) for second-line treatment of metastatic colorectal cancer (MCC): results of a multina- 
tional phase III trial (EFC10262-VELOUR) [abstract O-0024]. Oral presented at: ESMO 13th World Congress on Gastrointestinal Cancer; 2011 Jun 22-25; Barcelona, Spain. Ann Oncol 2011; 22(suppl 5);v18.

4. Martinsson-Niskanen T, Riisbro R, Larsson L, Winstedt L, Stenberg
Y, Pakola S, et al. Monoclonal antibody TB-403: a first-in-human, Phase I, double-blind, dose escalation study directed against placental growth factor in healthy male subjects. Clin Ther 2011;33: 1142-9. 